\title{
An fNIRS Research on Prefrontal Cortex Activity Response to Pleasant Taste
}

\author{
Chenghong $\mathrm{Hu}^{1}$, Yoshiko Kato ${ }^{2^{*}}$, Zhiwei Luo ${ }^{1}$ \\ ${ }^{1}$ Graduate School of System Informatics, Kobe University, Kobe, Japan \\ ${ }^{2}$ Graduate School of Human Development and Environment, Kobe University, Kobe, Japan \\ Email: *ykatou@crystal.kobe-u.ac.jp
}

Received November 11, 2013; revised December 11, 2013; accepted December 24, 2013

Copyright (C) 2013 Chenghong Hu et al. This is an open access article distributed under the Creative Commons Attribution License, which permits unrestricted use, distribution, and reproduction in any medium, provided the original work is properly cited.

\begin{abstract}
Functional Near-Infrared Spectroscopy (fNIRS), as a non-invasive neuroimaging technique, was used to monitor the activation of prefrontal lobe on human brain during sweet taste processing. The primary aim of the present study was to find the region of interest (ROI) which is related to sweetness, and make further understanding of the central organization of taste. Based on event-related design, the experiments were performed with 16 volunteers by sweet taste stimulus. It was confirmed that the prefrontal cortex (PFC) is involved in sweet taste processing and fNIRS provided an alternative way for studying taste-related brain function under more natural conditions. This study might be effective for detecting the accession area in the cortex of sweet taste and helpful for studying on human feeding and taste disease like taste dyspepsia or disorder.
\end{abstract}

Keywords: Near-Infrared Spectroscopy; Gustation; Sweet Taste; Prefrontal Cortex

\section{Introduction}

Recently, the prevalence of taste diseases which is caused by aging society and the changes of diet structure, such as anorexia nervosa, dyspepsia and taste disorder, appears to be increasing [1,2]. Although these diseases are not life-threatening diseases directly, they have a strong impact on our quality of life $[3,4]$. For this reason, the interest in understanding gustatory processing is growing, not only for basic science, but also for applications. Some knowledge and theory about high cognitive processes for taste have been accumulated. However, owing to the complexity of human brain and limitation of device, by now, most questions about the central organization of taste are still largely unanswered.

With the development of modern neuroimaging techniques such as positron emission tomography (PET), functional magnetic resonance imaging (fMRI) and functional near-infrared spectroscopy (fNIRS), a wider range of brain and gustatory researches has been accommodated. Among these less- or non-invasive neuroimaging techniques, we focused on fNIRS, which is widely used to monitor the brain activation and measure changes in the hemoglobin oxygenation state in human brain $[5,6]$.

${ }^{*}$ Corresponding author.
Compared to other neuroimaging methods, fNIRS as a new neuroimaging technique, which is completely noninvasive and does not require strict motion restriction, is desirable [7,8]. Meanwhile, it has been low cost. It enables brain studies on subjects who are difficult to examine with PET and fMRI physiologically and/or physically [9].

The prefrontal cortex (PFC), located in the very front of the brain, is in charge of abstract thinking and thought analysis and is also responsible for regulating behavior. Since the PFC regulated so many behavior and thoughtprocessing pathways, it is gradually drawing the researchers' attention. Recently, some cognitive process and sensory evaluation studies, using PET, MRI and another neuroimaging technique, have accumulated some knowledge and theories about visual and auditory modalities for PFC functions. Subsequently, the question whether the PFC is also involved in taste cognitive processing is widely assumed. Related to this question, previous studies found that the lateral prefrontal cortex is closely related to taste and other food-related activities [10-13]. Although some works have been done to examine the lateral PFC functions with taste, the results are not identical.

As mentioned above, in present study, we used fNIRS to monitor human PFC function during taste processing 
to find the region of interest (ROI) which is related to pleasant taste, and make further understanding of the central organization of taste. We also hope our findings will be helpful for studying on some taste diseases which are caused by aging and diet.

The paper is organized as follows: Section 2 presents the stimuli, experimental procedure and data analysis in details. The results of present study are shown in Section 3 , followed by discussion about the results in Section 4 . Finally, some conclusions are drawn in Section 5.

\section{Materials and Methods}

\subsection{Subjects}

16 healthy right-handed subjects took part in the study. The average age of the subjects was 26.3 (range from 20 to 44 year). Subjects refrained from eating for at least 2 hours prior to running the experiment. Before the present experiment, the subjects were told about the experiment and a pilot study was done. All subjects gave informed consent approved by the Human Ethics Committee of Health Promotion and Education Graduate School of Human Development and Environment in Kobe University.

\subsection{Stimuli}

Sweetened solution $(5 \%$ and $10 \%$ solution of sucrose dissolved in purified water) was used as tastant in this study, which is a signal of energy supply and usually brings a pleasurable sensation. Purified water (tasteless) was selected as a control condition to minimize the artificial noise from somatosensory effects, swallowing effects and other brain function. Samples were served at room temperature.

\subsection{Experimental Procedure}

During the fNIRS recording session, the subjects were seated in a comfortable chair in a quiet room. The eventrelated design was adopted for detecting hemodynamic response to gustatory stimuli. Figure 1 presented the schematic diagram of the protocol. The subjects were required to try to relax as possible for 40 seconds as baseline. Then stimulus, in quantities of $8 \mathrm{ml}$, was manually injected in to the subject's mouth via a syringe connected to a tube. And the subject was asked to hold the stimulus in mouth and taste for 25 seconds, which was defined as task. Finally, they spitted it out and rinsed his/her mouth with purified water to prevent adaptation. In this study, they tasted the purified water, $5 \%$ solution of sucrose and $10 \%$ solution of sucrose in order. Throughout the whole process, the subject kept closing eyes.

After the fNIRS sessions, the intensity of tastants was rated by the subjects as a subjective evaluation on the dislike-like.

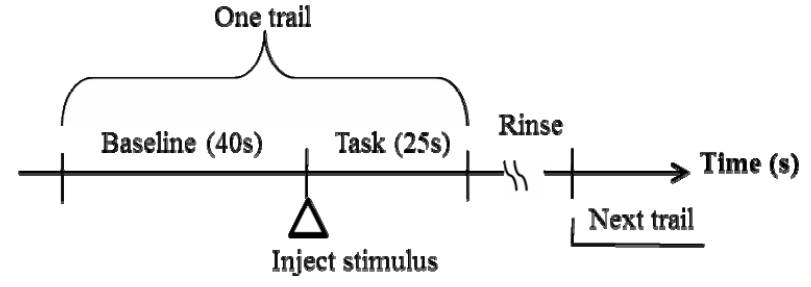

Figure 1. Experimental protocol.

\section{4. fNIRS and Data Analysis}

While the subject performed the tests, we recorded activity in the lateral PFC using a multichannel fNIRS sensor of OEG-16 (Spectratech Inc., Tokyo, Japan). Both 770 $\mathrm{nm}$ and $840 \mathrm{~nm}$ wavelengths were used to measure changes in oxyhemoglobin $(\triangle \mathrm{oxy} H \mathrm{H})$ and deoxyhemoglobin $(\Delta$ deoxyHb) concentration [14]. Subsequently, the total hemoglobin signal $(\Delta$ total $\mathrm{Hb})$ was calculated as a sum of $\triangle \mathrm{oxyHb}$ and $\Delta$ deoxyHb. The signal bandwidth of oxyHb and deoxyHb is $0.76 \mathrm{~Hz}$. As illustrated in Figure 2(a), in present study two arrays of six emitters and six detectors were paced ate alternate points and 16 measurement points were available (emitter-detector distance was 3 $\mathrm{cm})$. In order to have a reliable cerebral structural correlation, the center of the probe matrix was placed on the $\mathrm{Fz}$ of the international $10-20$ system. Since we conducted the experiment with the OEG-16 without any supplementary tool such as a $3 \mathrm{D}$ digitizer, it is difficult to identify the activated foci of human brain. To resolve this problem, we referred to the spatial registration of fNIRS device ETG4000 $3 \times 11$ (manufactured by Hitachi), which contained the channels of spectratech OEG-16 system. The probabilistic for each channel are listed in Table 1 as the standard MNI coordinates produced by Montreal Neurological Institute in Canada (Figure 2(b)).

Regarding the data analysis, since data from the detectors were too noisy for analysis, data were first filtered with moving average (data points: 5) to eliminated physiological noise. Baseline correction was performed using linear fitting. Following this, activation of baseline and task for each trail was averaged. To determine the significantly activated channels, we firstly compared the baselines and tasks to examine whether the channel is activated by tastants. Cognitive subtraction was used to compare purified water with sweetened solution so as to identify the true activities evoked by sweet taste backwardly. Statistical analyses were conducted using PAWS version 18.0 (SPSS Inc.).

\section{Result}

The signal changes in $\Delta \mathrm{oxyHb}, \Delta$ deoxyHb and $\Delta$ total $\mathrm{Hb}$ for each channel during tasting $5 \%$ sweetened solution were exhibited in Figure 3 and it seemed to almost nothing change. On the other hand, Figure 4 presented 
Right hemisphere I Left hemisphere

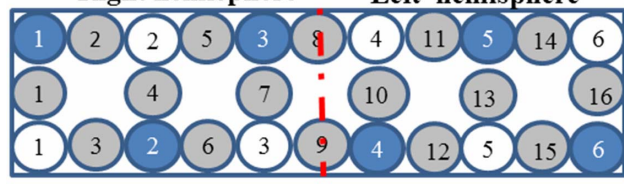

Position of light-emitting part (1-6)

Position of light-receiving part (1-6)

Measurement position(channels 1-16)

(a)

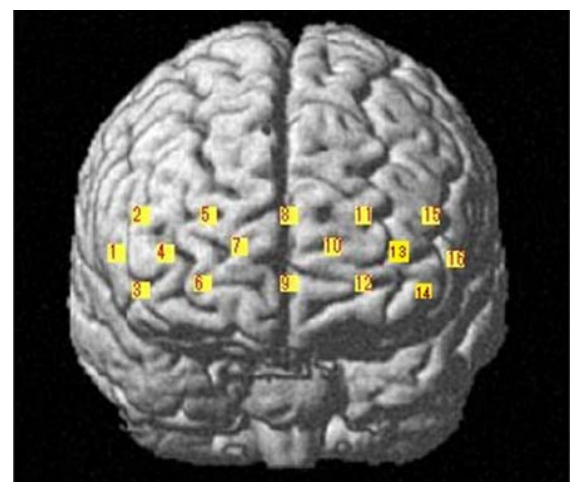

(b)

Figure 2. (a) Schematic illustration of the multi-channel array (Spectratech OEG-16 system, 16 channels). (b) fNIRS channels' estimated cortical location in MNI space.

the concentration changes of oxyHb, deoxyHb and totalHb in the $10 \%$ sweet solution processing and it showed a relative decrease of oxyHb, accompanied by a deoxyHb increase in most channels. In addition, the totalHb response pattern seemed to be similar to the oxyHb.

For statistical analysis, Analysis of variance (ANOVA) on the data was performed separately for $\triangle \mathrm{oxyHb}$, $\Delta$ deoxyHb and $\Delta$ totalHb. With the help of ANOVA, from the global data of 16 subjects, the results were obtained as following. The brain responses to sweet solution with different concentrations were seen in different anatomical area in PFC summarized in Table 2. Furthermore, the activated channels induced by the higher concentration were more than that induced by the lower concentration in present study. Specifically, for $\Delta \mathrm{oxyHb}$, the results of statistical analysis revealed no significant effect of PFC except $\mathrm{Ch} 7$ in the lower concentration condition, which is closed to frontopolar area and orbitofrontal area. While the in the higher concentration condition the following nine channels revealed significance located in bilateral pars triangularis Broca's area (Ch1and Ch16), dorsolateral PFC (Ch3, Ch4, Ch11, Ch13 and Ch16), frontopolar area (Ch4,Ch10,Ch11, $\mathrm{Ch} 13)$, and the orbitofrontal area (Ch4, Ch6, Ch10, Ch12 and Ch13). For $\Delta$ deoxyHb, two channels (Ch4 and Ch6) showed significance for lower concentration solution referred to frontopolar area, dorsolateral PFC and orbi tofrontal area in the left hemisphere, while significance
Table 1. Probabilistic estimation of channels' cortical location in MNI coordination and corresponding anatomical label.

\begin{tabular}{|c|c|c|c|c|c|}
\hline \multirow{3}{*}{$\mathrm{ch}$} & \multicolumn{4}{|c|}{ MNI coordinate } & \multirow{3}{*}{$\begin{array}{l}\text {-Anatomical label } \\
\text { BA }\end{array}$} \\
\hline & \multicolumn{3}{|c|}{ Position } & \multirow[t]{2}{*}{$\mathrm{SD}$} & \\
\hline & $\mathrm{x}$ & $\mathrm{y}$ & z & & \\
\hline 1 & 53 & 39 & 7 & 5.97 & $\begin{array}{l}45 \text { - pars triangularis Broca's area } \\
46 \text { - Dorsolateral PFC }\end{array}$ \\
\hline 2 & 46 & 48 & 20 & 6.24 & $\begin{array}{l}\text { 46- Dorsolateral PFC } \\
45 \text { - pars triangularis Broca's area }\end{array}$ \\
\hline 3 & 46 & 54 & -5 & 5.11 & 46 - Dorsolateral PFC \\
\hline 4 & 38 & 60 & 8 & 5.07 & $\begin{array}{l}10 \text { - Frontopolar area } \\
46 \text { - Dorsolateral PFC } \\
11 \text { - Orbitofrontal area }\end{array}$ \\
\hline 5 & 25 & 65 & 20 & 5.75 & $\begin{array}{l}10 \text { - Frontopolar area } \\
46 \text { - Dorsolateral PFC }\end{array}$ \\
\hline 6 & 27 & 68 & -3 & 4.50 & 11 - Orbitofrontal area \\
\hline 7 & 15 & 70 & 9 & 5.03 & $\begin{array}{l}10 \text { - Frontopolar area } \\
11 \text { - Orbitofrontal area }\end{array}$ \\
\hline 8 & 3 & 66 & 20 & 7.07 & 10 - Frontopolar area \\
\hline 9 & 3 & 68 & -3 & 5.54 & $\begin{array}{l}10 \text { - Frontopolar area } \\
11 \text { - Orbitofrontal area }\end{array}$ \\
\hline 10 & -13 & 70 & 9 & 4.91 & $\begin{array}{l}10 \text { - Frontopolar area } \\
11 \text { - Orbitofrontal area }\end{array}$ \\
\hline 11 & -24 & 65 & 20 & 5.83 & $\begin{array}{l}10 \text { - Frontopolar area } \\
46 \text { - Dorsolateral PFC }\end{array}$ \\
\hline 12 & -23 & 70 & -3 & 4.64 & 11 - Orbitofrontal area \\
\hline 13 & -35 & 55 & 22 & 5.12 & $\begin{array}{l}10 \text { - Frontopolar area } \\
11 \text { - Orbitofrontal area } \\
46 \text { - Dorsolateral PFC }\end{array}$ \\
\hline 14 & -44 & 49 & 20 & 6.46 & $\begin{array}{l}45 \text { - pars triangularis Broca's area } \\
46 \text { - Dorsolateral PFC }\end{array}$ \\
\hline 15 & -43 & 55 & -5 & 4.95 & $\begin{array}{l}10 \text { - Frontopolar area } \\
46 \text { - Dorsolateral PFC }\end{array}$ \\
\hline 16 & -51 & 40 & 6 & 6.31 & $\begin{array}{l}45 \text { - pars triangularis Broca's area } \\
46 \text { - Dorsolateral PFC }\end{array}$ \\
\hline
\end{tabular}

ch: cannel; PFC: prefrontal cortex.

Table 2. Activated channels of activated channels of $\Delta 0 x y H b, \Delta$ deoxyHb and $\Delta$ totalHb analysis.

\begin{tabular}{|c|c|c|}
\hline & $5 \%$ solution of sucrose & $10 \%$ solution of sucrose \\
\hline$\Delta \mathrm{oxyHb}$ & Ch no. 7 & $\begin{array}{crrrrr}\text { Ch no. } 1 & 3 & 4 & 6 & 10 & 11 \\
12 & 13 & 16 & & \end{array}$ \\
\hline$\Delta$ deoxyHb & Chno. 46 & Ch no. $2 \quad 3 \quad 5 \quad 16$ \\
\hline$\Delta$ totalHb & Ch no. 7 & $\begin{array}{llllll}\text { Ch no. } 4 & 6 & 12 & 13 & 15 & 16\end{array}$ \\
\hline
\end{tabular}

Channels that exhibited significant changes in $\triangle \mathrm{oxyHb}, \Delta$ deoxyHb and $\Delta$ totalHb are listed for the two sweetened solution with different concentration. The results represented the group analysis for 16 subjects.

could be found in bilateral dorsolateral PFC, pars triangularis Broca's area (Ch2, Ch3, Ch5, and Ch16) for the higher one. For $\Delta$ totalHb, $\mathrm{Ch} 7$ also showed a significant change for the lower concentration, and six channels (Ch4, Ch6, Ch12, Ch13, Ch15, and Ch16) showed significant changes for the higher one. In addition, the individual analysis showed that the activated areas of brain 


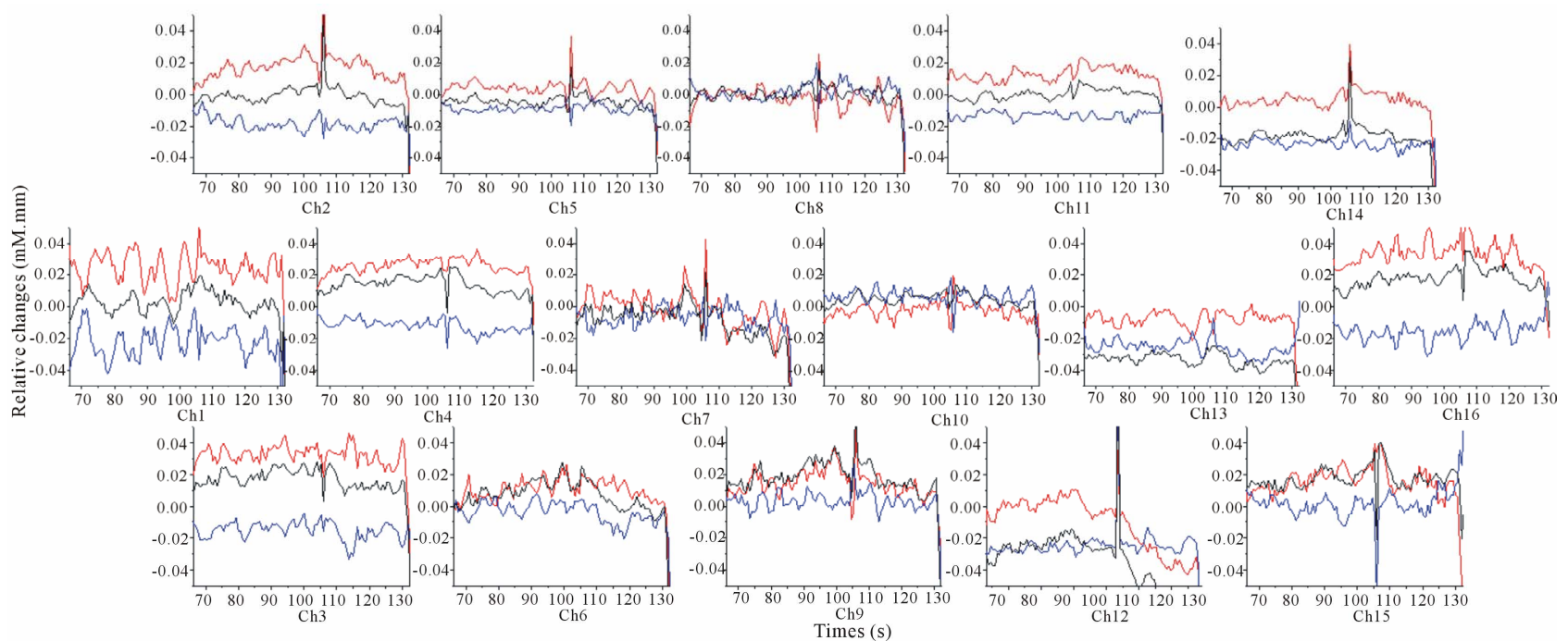

Figure 3. Grand average (16 subjects) concentration changes of oxyHb, deoxyHb and totalHb during $5 \%$ sweetened solution of sucrose. (Hemoglobin concentration changes are exhibited in colors lines: $\Delta$ oxyHb, red; $\Delta d e o x y H b$, blue; $\Delta$ totalHb, black).

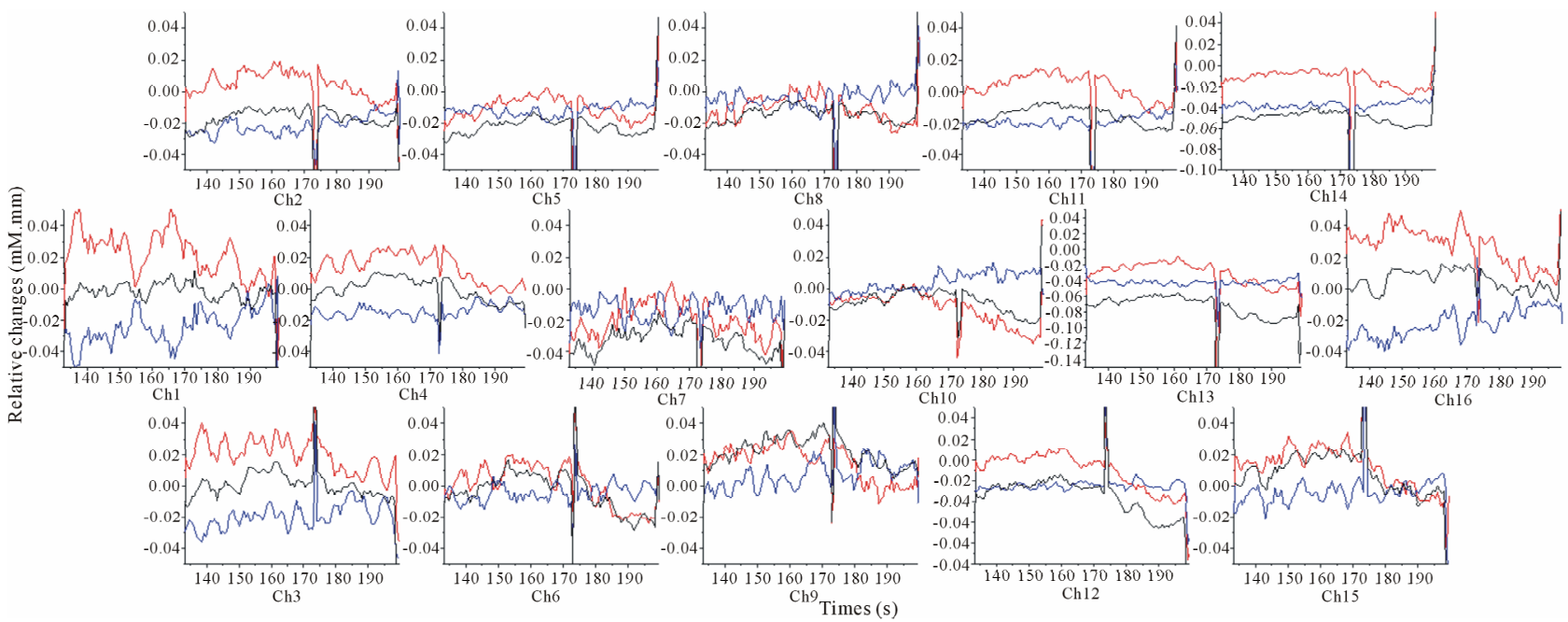

Figure 4. Grand average (16 subjects) concentration changes of oxyHb, deoxyHb and totalHb during $10 \%$ sweetened solution of sucrose. (Hemoglobin concentration changes are exhibited in colors lines: $\Delta$ oxyHb, red; $\Delta$ deoxyHb, blue; $\Delta$ totalHb, black).

are different among all of the subjects under the sweet stimuli processing.

\section{Discussion}

Some traditional neurophysiology and neuro-anatomy studies have accumulated a lot of knowledge and theories about taste organ, anatomic constitution of taste pathways, transduction of taste signal and classification of tastants. Most of them depended on autopsy, head injured patients and animal experiments. The accumulated knowledge, in particular to taste-related brain function, is far from complete. With the advent of modern neuroimaging techniques, a wider range of taste-related brain function can be monitored more objectively and effectively. Some studies have shown that the neuron activity is closely re- lated to cell energy metabolism. While the functional area of human brain is stimulated and activated under some task condition, regional brain activation is accompanied by increases in regional cerebral blood flow (rCBF) and the regional cerebral oxygen metabolic rate (rCMRO2). It is widely accepted that the degree of the increases in rCBF exceeds that of the increase in $\mathrm{rCMRO} 2$ [15], which results in a decrease in deoxyHb in venous blood. The fNIRS can measures the concentration changes of oxyHb, $\mathrm{deoxyHb}$ and totalHb and to reveal the brain activation. Owing its strengths and advantages, fNIRS provide an alternative way to monitor taste-related brain function. The present study gave evidence that the fNIRS can be used to monitor the human prefrontal cortex activity which is respond to sweet taste. 
In previous studies, two authorized models utilized in cognitive experiments using fMRI are a block design model and an event-related design model. The experiments using the block design are simpler to implement because careful randomization and spacing of different stimulus categories is not required [16]. However, in a block design, two or more conditions are alternated in blocks in some order, and this makes the type of stimulus within each block very predictable, which will interfere with experiment result as a consequence. Relatively, the latter event-related design is more complex and less selected for fMRI studies. It permits a randomized presentation of stimuli that theoretically reduce confounds arising from stimulus predictability. So far, many researches usually utilized the block design to do the fMRI experiments for investigating the taste-related brain function. And some previous studies suggested that when some taste rewards are predictable, brain regions recruited during expectation are, in part, dissociable from areas responding to reward receipt [17]. Therefore, in present study, the experiment performed on event-related design so that the interference arising from stimulus predictability could be avoided effectively.

To date, the issue of cortex localization of taste cognitive processing is still on dispute. In nonhuman primate, behavioral, ethological and electrophysiological evidence suggested that the sense of taste is conveyed via nucleus of the solitary tract, thalamus, projected to the primary gustatory cortex (anterior insula/ frontal operculum), and then further processed in the second gustatory cortex which is sometimes referred to orbitofrontal cortex in frontal lobe [18]. Furthermore, in humans, some experiments for investigating gustatory neural circuitry also have been done among human and all most of them supported the issue above and indicated these areas presented above are activated by taste stimuli [19-22]. Especially relevant to sweet taste, severed as a signal of energy regulation [23] and usually bringing a pleasurable sensation, some evidences suggested glucose ingestion could improve cognition [24,25] and also have beneficial effects on attentional control $[26,27]$. Furthermore, considering its importance, few studies have used EEG, PET, fMRI or another neurophysiology and neuroimaging techniques to examine brain activation pattern associated with sweet taste. For instance, evidences for EEG suggests that clear right front central activation and caudolateral orbitofrontal cortex was specifically observed for sweet taste [28], while other fMRI or PET studies found increased rCBF values in the thalamus, the insular cortex, the anterior cingulate gyms, the parahippocampal gyrus, the lingual gyrus, the caudate nucleus, and the temporal gyri, especially in the left or bilateral hemisphere $[11,20]$. To summary, these studies reported above suggested that sweet taste processing acutely influenced the cerebral ac- tivity. However, the mechanism for sweet gustatory system is still uncompleted. Hence, our study used fNIRS, a new noninvasive neuroimaging technique, to investigate the sweet taste cognitive mechanism and results suggested the bilateral prefrontal cortex was activated in taste system. To exact, parts of pars triangularis Broca's area, dorsolateral PFC, frontopolar area, and the orbitofrontal area was acutely influenced when the participants were in the sweet taste condition. Significant activation in orbitofrontal cortex with subjective pleasantness is consistent with an important role for the orbitofrontal cortex in human emotion and motivation. The more regions reported here is similar to some previous findings but also shows some differences for different experimental designs or tasks [11-13]. Admittedly, this study has some limitations and more evidence is required to clarify this issue. A future NIRS study may overcome the influences induced by subjects' number, taste concentration, persons' physical self [29] or another factors, to further assesse questions about taste cortical activation on human brain.

\section{Conclusion}

Taken together, fNIRS, a noninvasive neuroimaging technique, created potential for studying a wider variety of taste-related brain function under more natural conditions. The present study demonstrated that the LPFC is involved in representing the subjective pleasantness of tastants, which may be important in influencing whether food is selected as a goal of action. We also hope the investigation will further contribute to the understanding of sweet taste cognitive mechanisms and even to provide some theoretical evidence for studying on taste dyspepsia and disorder.

\section{Acknowledgements}

We thank the volunteers who participated in this study. This work was supported by Grant-in-Aid for Scientific Research (C) 23500945 from the Japan Society for the Promotion of Science.

\section{REFERENCES}

[1] Y. Kato, "Relationship among Stress, Drive for Thinness, and Eating Behavior of Female University Students: Attitude toward Sweetness an Inclination to Eating Disorders," Japan Society of Home Economics, Vol. 55, No. 8, 2007, pp. 453-461.

http://dx.doi.org/10.1196/annals.1396.028

[2] M. D. Parrott and C. E. Greenwood, "Dietary Influences on Cognitive Function with Aging: From High-Fat Diets to Healthful Eating," Annals of the New York Academy of Sciences, Vol. 1114, 2007, pp. 389-397.

[3] H. C. Liu, "Study of Relationship between Oral Function and Brain Function," Chinese Journal of Prosthodontics, 
Vol. 3, 2002, pp. 137-139.

[4] A. Drewnowski, "Taste Preference and Food Intake," Annual Review of Nutrition, Vol. 17, 1997, pp. 237-253. http://dx.doi.org/10.1146/annurev.nutr.17.1.237

[5] F. F. Jobsis, "Noninvasive Infrared Monitoring of Cerebral and Myocardial Oxygen Sufficiency and Circulatory Parameters," Science, Vol. 198, 1977, pp. 1264-1267. http://dx.doi.org/10.1126/science.929199

[6] L. Gagnon, M. A. Yucel, M. Dehaes, R. J. Cooper, K. L. Perdue, J. Selb, et al., "Quantification of the Cortical Contribution to the NIRS Signal over the Motor Cortex Using Concurrent NIRS-fMRI Measurements," NeuroImage, Vol. 59, No. 4, 2012, pp. 3933-3940. http://dx.doi.org/10.1016/j.neuroimage.2011.10.054

[7] S. Coyle, T. Ward, C. Markham and G. McDarby, "On the Suitability of Near-Infrared Systems for Next Generation Brain Computer Interfaces," Physiological Measurement, Vol. 25, No. 4, 2004, pp. 815-822. http://dx.doi.org/10.1088/0967-3334/25/4/003

[8] Y. Hoshi, N. Kobayashi and M. Tamura, "Interpretation of Near-Infrared Spectroscopy Signals: A Study with a Newly Developed Perfused Rat Brain Model," Journal of Applied Physiology, Vol. 90, No. 5, 2001, pp. 16571662.

[9] Y. Hoshi, "Functional Near-Infrared Optical Imaging: Utility and limitation in Human Brain Mapping," Psychophysiology, Vol. 40, No. 4, 2003, pp. 511-520. http://dx.doi.org/10.1111/1469-8986.00053

[10] E. T. Rolls, Z. J. Sienkiewicz and S. Yaxley, "Hunger Modulates the Responses Togustatory Stimuli of Single Neurons in the Caudolateral Orbitofrontal Cortex of the Macaque Monkey," European Journal of Neuroscience, Vol. 1, No. 1, 1989, pp. 53-60. http://dx.doi.org/10.1111/j.1460-9568.1989.tb00774.x

[11] M. L. Kringelbach, I. E. de Araujo and E. T. Rolls, "Taste-Related Activity in the Human Dorsolateral Prefrontal Cortex," NeuroImage, Vol. 21, No. 2, 2004, pp. 781-788. http://dx.doi.org/10.1016/j.neuroimage.2003.09.063

[12] M. Okamoto, H. Dan, A. K. Singh, F. Hayakawa, V. Jurcak, T. Suzuki, K. Kohyama and I. Dan, "Prefrontal Activity during Flavor Difference Test: Application of Functional Near-Infrared Spectroscopy to Sensory Evaluation Studies," Appetite, Vol. 47, No. 2, 2006, pp. 220-232. http://dx.doi.org/10.1016/j.appet.2006.04.003

[13] M. Okamoto, M. Matsunami, H. Dan, T. Kohata, K. Kohyama and I. Dan, "Prefrontal Activity during Taste Encoding: An fNIRS Study," NeuroImage, Vol. 31, No. 2, 2006b, pp. 796-806. http://dx.doi.org/10.1016/i.neuroimage.2005.12.021

[14] D. T. Delpy, M. Cope, P. van der Zee, S. Arridge, S. Wray and J. Wyatt, "Estimation of Optical Pathlength through Tissue from Direct Time of Flight Measurement," Physics in Medicine and Biology, Vol. 33, No. 12, 1988, pp. 1433-1442.

http://dx.doi.org/10.1088/0031-9155/33/12/008

[15] P. Fox and M. E. Raichle, "Focal Physiological Uncoupling of Cerebral Blood Flow and Oxidative Metabolism during Somatosensory Stimulation in Human Subjects," Proceedings of the National Academy of Sciences of the United States of America, Vol. 83, No. 4, 1986, pp. 11401144. http://dx.doi.org/10.1073/pnas.83.4.1140

[16] D. I. Donaldson and R. L. Buckner, "Effective Paradigm Design," In: S. M. Smith, Ed., Functional MRI: An Introduction to Methods, Oxford University Press, Oxford, 2001, pp. 177-196.

[17] J. P. O’Doherty, R. Deichmann, H. D. Critchley and R. J. Dolan, "Neural Responses during Anticipation of a Primary Taste Reward," Neuron, Vol. 33, No. 5, 2002, pp. 815-826. http://dx.doi.org/10.1016/S0896-6273(02)00603-7

[18] D. H. Zald, M. C. Hagen and J. V. Pardo, "Neural Correlates of Tasting Concentrated Quinine and Sugar Solutions," Journal of Neurophysiology, Vol. 87, No. 2, 2002, pp. 1068-1075.

[19] M. A. Barry, J. C. Gatenby, J. D. Zeiger and J. C. Gore, "Hemispheric Dominance of Cortical Activity Evoked by Focal Electrogustatory Stimuli," Chemical Senses, Vol. 26, No. 56, 2001, pp. 471-482. http://dx.doi.org/10.1093/chemse/26.5.471

[20] S. Kinomura, R. Kawashima, K. Yamada, S. Ono, M. Itoh, S. Yoshioka, et al., "Functional Anatomy of Taste Perception in the Human Brain Studied with Positron Emission Tomography," Brain Research, Vol. 659, No. 1-2, 1994, pp. 263-266. http://dx.doi.org/10.1016/0006-8993(94)90890-7

[21] T. C. Pritchard, D. A. Macaluso and P. J. Eslinger, "Taste Perception in Patients with Insular Cortex Lesions," $\mathrm{Be}$ havioral Neuroscience, Vol. 113, No. 4, 1999, pp. $663-$ 671. http://dx.doi.org/10.1037/0735-7044.113.4.663

[22] R. J. Zatorre and M. Jones-Gotman, "Functional Imaging of the Chemical Senses," In: A. W. Toga and J. C. Mazzoiotta, Eds., Brain Mapping: The Systems, Academic Press, San Diego, 2000, pp. 403-424. http://dx.doi.org/10.1016/B978-012692545-6/50015-5

[23] S. E .Swithers and T. L. Davidson, "A Role for Sweet taste: Calorie Predictive Relations in Energy Regulation by Rats," Behavioral Neuroscience, Vol. 122, No. 1, 2008, pp. 161-173. http://dx.doi.org/10.1037/0735-7044.122.1.161

[24] L. M. Riby, A. Meikle and C. Glover, "The Effects of Age, Glucose Ingestion and Gluco-Regulatory Control on Episodic Memory," Age Ageing, Vol. 33, No. 5, 2004, pp. 483-487. http://dx.doi.org/10.1093/ageing/afh173

[25] L. M. Riby, S. I. Sunram-Lea, C. Graham, J. K. Foster, T. Cooper, C. Moodie, et al., "P3b Versus P3a: An EventRelated Potential Investigation of the Glucose Facilitation Effect," Journal of Psychopharmacology, Vol. 22, No. 5, 2008, pp. 486-492.

http://dx.doi.org/10.1177/0269881107081561

[26] A. B. Scholey, S. I. Sunram-Lea, J. Greer, J. Elliott and D. O. Kennedy, "Glucose Administration Prior to a Divided Attention Task Improves Tracking Performance But Not Word Recognition: Evidence against Differential Memory Enhancement," Psychopharmacology (Berl), Vol. 202, 2009, pp. 549-558. http://dx.doi.org/10.1007/s00213-008-1387-1 
[27] C. Gagnon, C. E. Greenwood and L. Bherer, "The Acute Effects of Glucose Ingestion on Attentional Control in Fasting Healthy Older Adults," Psychopharmacology (Berl), Vol. 211, No. 3, 2010, pp. 337-346.

http://dx.doi.org/10.1007/s00213-010-1905-9

[28] I. H. A. Franken, J. Huijding, I. M. T. Nijs and J. W. van Strein, "Electrophysiology of Appetitive Taste and Appe- titive Taste Conditioning in Humans," Biological Psychology, Vol. 86, No. 3, 2011, pp. 273-278.

http://dx.doi.org/10.1016/j.biopsycho.2010.12.008

[29] J. R. Ganchrow and R. P. Erickson, "Neural Correlates of Gustatory Intensity and Quality," Neurophysiology, Vol. 33, 1970, pp. 768-783. 